\title{
Chassis for future mobility: challenges and solutions
}

Nico Rosberg, TRE GmbH, F1 World Champion 2016

This manuscript is not available according to publishing restriction.

Thank you for your understanding. 